\title{
A dictionary of Astronomy for the French Sign Language (LSF)
}

\author{
Dominique Proust ${ }^{1}$, Daniel Abbou ${ }^{2}$ and Nasro $\mathrm{Chab}^{3}$ \\ ${ }^{1}$ Observatoire de Paris-Meudon, \\ 92195 Meudon Principal Cedex, France \\ email: dominique.proust@obspm.fr \\ ${ }^{2}$ Groupe France-Television, France 5 \\ 7 Esplanade Henri-de-France, 75907 Paris, France \\ email: daniel.abbou@neuf.fr \\ ${ }^{3}$ Musée des Arts et Métiers \\ 292 rue Saint Martin \\ 75003 Paris, France \\ email: nasreddine.chab@cnam.fr
}

\begin{abstract}
Since a few years, the french deaf communauty have access to astronomy at ParisMeudon observatory through a specific teaching adapted from the French Sign Language (Langue des Signes Française, LSF) including direct observations with the observatory telescopes. From this experience, an encyclopedic dictionary of astronomy The Hands in the Stars is now available, containing more than 200 astronomical concepts. Many of them did not existed in Sign Language and can be now fully expressed and explained.
\end{abstract}

Keywords. astronomy, teaching, deafness

\section{Take the stars by the hand}

I am not deaf, just a little hard of hearing! This comment by the famous Professor Tournesol sets down a marker in Hergés work and it makes one wonder how, in the context of education which is not much inclined to accept deafness, Tournesol could have followed a scientific path which resulted in his professorship. In a world where teaching is almost exclusively directed at pupils and students who can hear, it is difficult to imagine the young Tryphon Tournesol learning ballistics or nuclear physics without any problem. He probably wasnt deaf from birth and unless he had been force-fed some form of speech therapy, his speaking voice was sufficiently clear to be understood by those around him, even those as inattentive as the $\operatorname{Dupon}(\mathrm{d})(\mathrm{t}) \mathrm{s}$. Note also that Haddocks name was constantly being screeched by Bianca Castafiore at the top of her voice which surely indicates that she too had a hearing problem particularly in the lower ranges of the audio spectrum (Haddock was probably a bass-baritone, doubtless helped along by a somewhat large consumption of whisky), but this didnt stop her reaching a top C sharp in the Jewel Song from Gounods Faust. However, moving beyond the fictional world of Tintin, a society that looks after its minorities has to make its scientific culture available to all, even to the deaf community.

In the scientific world in general, and astronomy in particular, Sign Language has been able to establish communication in a remarkable manner by its knowledge of deaf culture and suitable semiotics - the study of signs - which removes barriers and offers a dialogue between the deaf and the hearing population. 


\section{A dialogue of the deaf}

The sign language has its own vocabulary and grammar and expresses itself in all manners of communication. However, unlike Sweden, where there is complete cultural integration, France has, on this point, been way behind intellectually. In an historical context, the deaf have been marginalised and kept apart by most civilisations, and information concerning them has only come down to us on rare occasions. In spite of the persistent legend that attributes the origin of a structured sign language to the Abbé de l'Épée (Charles-Michel, 1712-1789), it is very obvious that conveying ideas by signing existed long before among groups of deaf people in the same way that enclosed religious orders such as the Trappists respected the rule of silence or even the Indian tribes that used the body to communicate over a distance.

After the Abbé de lÉpée, LSF suffered many setbacks in its history. Father Sicard (1742-1822), the first director of the National Institute for Deaf-Mutes founded after the Abbé de l'Épée s death, escaped the guillotine at the hands of the revolutionary Tribunal in 1793 thanks mainly to pressure brought by his deaf pupils demonstrating in support of him. Above all, it was Bebian (1789-1839) who was responsible for the creation of true bilingualism at the Royal Institute for Deaf-Mutes. However, two schools of thought began to oppose each other, the French maintaining the tradition of gestures whilst a new tendency came from Leipzig which relied on teaching words and lip reading. Ferdinand Berthier (1803-1887), the most renowned of the deaf professors at the Institut de Paris, and himself very deaf, vehemently defended sign language when Jules Ferrys (french minister of education) introduced his education laws which introduced a uniformity of teaching, accentuated by a belief in positivism and anti-clericalism that promoted the idea that man, by his nature, could solve any problem that came his way, including deafness. Because of these different pressures and also in the context of growing industrialisation which could resolve all sorts of problems technically, such as hearing aids, sign language slowly but surely began to disappear in France. Nevertheless, Victor Hugo wrote in a letter dated 12 November 1845 to Berthier: What does deafness matter as along as the spirit can hear? The only, true, incurable deafness is that of intelligence. Meanwhile, sign language was progressing by leaps and bounds in the USA and Canada where it had been exported in 1816 by Laurent Clerc, a professor of the Institut de Paris.

The death-knell for sign language was first of all sounded at the Exposition Universelle (Universal Exhibition) in Paris in 1878 where an army of oral teachers tore to pieces all practical and cultural achievements; this act was ratified by the Milan Congress in 1880. This movement was supported by the Church and the middle class who were fiercely opposed to unsettling gestures: dont point, it!. Besides, the miniaturisation of hearing aids and the advent of speech therapy naturally gave a ready excuse to hide the deficiencies of individuals who had been carefully kept out of the mainstream of society. So, to cultural and socio-linguistic differences were added conformity by force and a whole community was reduced to silence by law. The single-mindedness of the Milan Congress in rejecting all sign language reflected centuries of religious and social prejudice. As an example, the famous adage masturbation makes you deaf, invented by the Lausanne doctor, Auguste Tissot (1739-1797), who was anxious to teach his patients a moral code based on the intransigence of fundamental Calvinism, was top of the list of misdeeds which made the deaf community even more blameworthy. In this way, the implementation of rigid and arbitrary social codes made it possible to impose frustrating and senseless rules on dozens of generations in order to put a moral terrorism in place.

In 1887, the last deaf professors were forced to retire during a memorable ceremony in which the new director of the Institution for deaf-mutes made a clever speech: Today, 
the mime will leave this Institution, never to return and speech will reign hereafter by itself. Inevitably, the consequence of these measures was a rapid deterioration of communication. To perpetuate triumphant oralism, teachers used all kinds of stratagems, in particular punishing any attempt at hand movements and this situation lasted until the 1970s. This disappearance of the sign language at the hands of oralism had some dramatic consequences, not only in France but throughout Europe. Numerous homophonic words cannot be understood by a deaf interlocutor, especially if the speaker has an accent, does not articulate or has a moustache. Very rapidly, oralism in France brought on a cultural desert for the deaf community just as modern maths did for generations of french school children in the 1960s.

Authoritarian regimes have always looked to rid themselves of minorities in general and the deaf in particular in the name of eugenics; thus the Nazis sterilized several tens of thousands of deaf women in Germany between 1933 and 1945. This isolation of the deaf community gave rise to a normal reaction in the face of oppression: a resistance movement. In particular, a desire to communicate between the young happens naturally by signing. There are, then, two forms of expression, one that needs to have a disciplined oralism in order to be able to respond to the structural demands of officialdom while the other is free, made by signing and often updated. Many deaf people who knew this era are sad witnesses today to the cultural desert that they experienced over many years. Some intellectuals rose up against this ostracism, in particular Henri Gaillard (1866-1939), a journalist and publisher of the Gazette des Sourds-Muets (The Deaf-Mute Gazette) who stood up for LSF. In 1924, the first Olympic Games for the deaf took place and in 1926, the Salon for Silent Artists was created. The majority of the deaf could however only hope of passing the level of a CAP (Certificate of Professional Competence) and the number of graduates could be counted on the fingers of one hand. The rate of unemployment rose to $30 \%$ chiefly among the most hard of hearing.

However, sign language didnt completely disappear everywhere since, from 1817, it started to develop in the Anglo-Saxon countries, and most notably by the creation of the Hartford Institute by Thomas-Hopkins Gallaudet (1787-1851) and Laurent Clerc (17851869). Little by little, France discovered with what success the deaf were integrated into the mainstream in the USA, Canada, Great Britain and Sweden. At the end of 1970, the phrase French sign language and its initials $L S F$ were introduced by the sociologist Bernard Mottez and in 1973, the National Union for the Social Integration for the Hard of Hearing shook up the political classes and finally got some results, in particular the translation of the televised news. However one had to wait until 1977 when, under much pressure and with the success obtained overseas, the Minister for Health withdrew the prohibition of the LSF and in 1991 for the National Assembly to allow the education of children by sign language. Nowadays, LSF has finally obtained its status as a complete language. It is taught in every region (with variants that correspond with regional accents) even though the oralist bastions still exist, most notably in the medical world where you can still find aficionados of the cochlear implant in spite of the trauma and post-operative risks involved.

LSF continues to make itself steadily known in an attempt to regain its status as a language in its own right. There is still however much resistance to deafness particularly in the area of administrative proceedings, the judiciary, medicine etc. It is even more a matter of regret that with only thirty hours of training, it is possibly to have a dialogue with a deaf person on general matters. In the scientific world it is interesting to see how easily communication can be effected. The examples of the Museum of Arts and Crafts and the City of Science at La Villette (both in Paris) are eye openers with the main exhibitions and conferences being signed by deaf but highly competent scientists. 


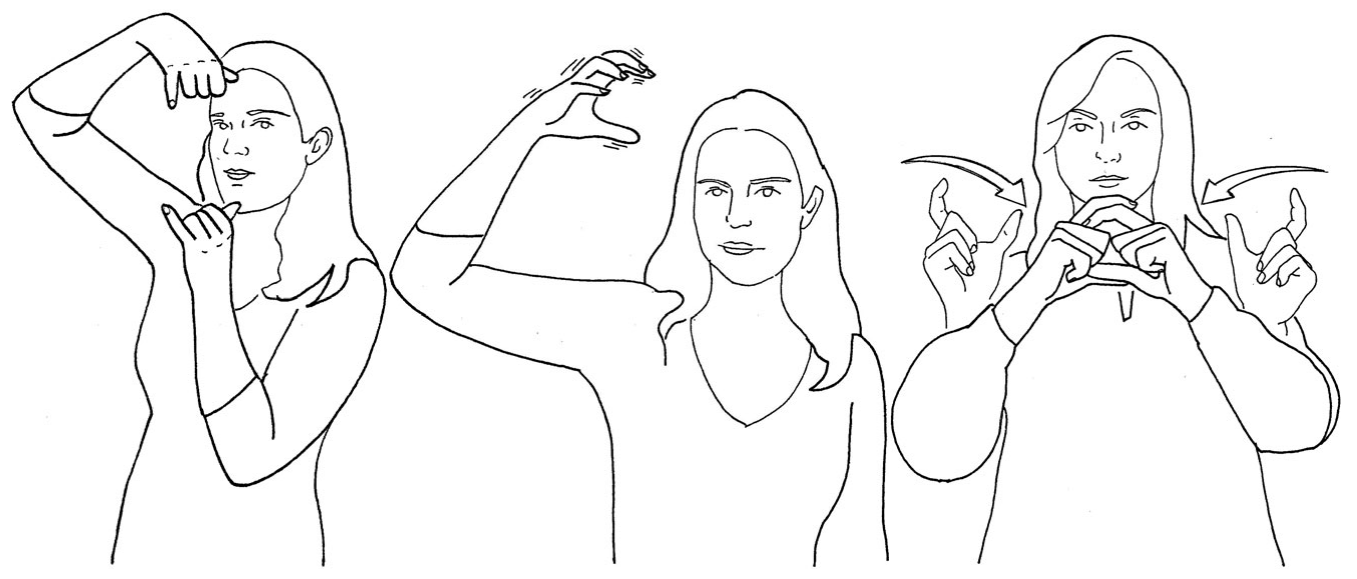

Figure 1. From left to right, how to sign Moon, Sun, Eclipse.

\section{Listening to famous deaf people}

The worlds of letters, arts and science have been much honoured by the deaf. Among the best know are: Pierre de Ronsard (1524-1585) who dedicated his sonnets to Cassandra, Marie and Helen, but who would have been hard pressed to respond to their call; Francisco Goya (1748-1828), one of the greatest ever painters but one who never had the pleasure of hearing the critics of his paintings; and finally, Beethoven (1770-1827) who only heard the Ode to Joy from the Ninth Symphony and his final string quartets in his own head.

In the world of science, Joseph Sauveur (1653-1716), a French mathematician and physician, and professor at the Collège de France in 1686, had been deaf from birth. In spite of his short life, John Goodricke (1767-1786) was a deaf astronomer who had a brilliant career. His observation of variable stars such as Algol in the constellation of Perseus, $\beta$ Lyre and $\delta$ Cephei allowed him to show the existence of the Cepheid family which are giant cold stars whose periodic pulses along with their intrinsic luminosity make them particularly useful as calibrators of distance as shown by Henrietta Leavitt (1868-1921) at Harvard College Observatory; Henrietta Leavitt became deaf at the age of 25. Two of the greatest inventions were made by people linked to deafness. Alexander Graham Bell (1847-1922) grew up in a family with a deaf mother and a father who perfected a system of visual language which translated sounds by symbols. As a professor in Boston working for deaf children and having himself a wife who was deaf, Bell developed a number of means of allowing communication between the deaf and the hearing, of which the most famous is the telephone (1877). The second is Thomas Edison (1847-1931) who only had $10 \%$ hearing in one ear. We owe him the invention of a process for recording and sound reproduction (the gramophone), as well as the first cinema projectors, the incandescent bulb and the improvement of telegraphy. The Edison Effect is known as the emission of electrons by heated metals.

\section{Science signs}

Sign language is a perfectly structured language with its own vocabulary and grammar and which follows precise rules which are linked with basic bodily expressions. Like all languages, it is always evolving and the scientific and technical vocabulary is always being brought up to date with new signs such as Number, Internet, DVD, Microprocessor, etc. 

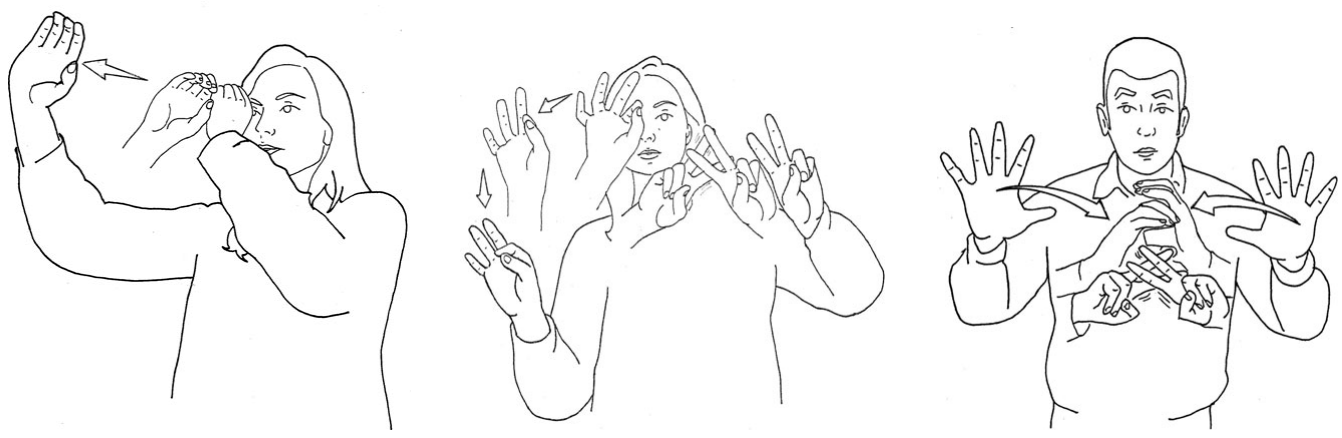

Figure 2. From left to right, how to sign Astronomy, Constellation, Globular cluster.

In mathematics, numbers are linked together by a sequence of signs; 1515 is signed as ONE THOUSAND + FIVE HUNDRED + FIFTEEN. Big numbers (millions, billions) have their own signs and operators. As an example, the square root sign $[\sqrt{ }]$ is signed in an identical fashion with the hands. All quantities are signed whether weight, surface, volume or distance. Pythagoras's theorem is stated in the same way as its oral version in that the hypotenuse is signed as the side opposite the right angle. Geometry is signed in an identical way with the hands first of all depicting a perpendicular, a level or an area. You can also indicate with accuracy the source of a system of coordinates.

Physics is made up of lots of very explicit signs for each property. Constants are designated by the same letters: $c$ is the speed of light (SPEED + LIGHT) with $c$ equal to $300000 \mathrm{~km}$ per second. ELECTRICITY is signed with the fists facing the signer with the index fingers bent upwards to indicate the electrodes. NUCLEAR ENERGY is made up of two signs, the first being the generic sign for all forms of energy and the second symbolising nuclear power. In Chemistry, elements are signed either specifically or by their symbol.

Astronomy is one of the areas in which expression in sign language can be both exact and poetic. The signs attributed to the different planets in the solar system relate to their own characteristics. Mercury is very close to the Sun, Mars is red, Jupiter is represented by its well know red spot which has been seen by telescopes for more than a century and Saturn is indicated by its ring. The representation of the sky is simplified since the majority of the constellations evoke animals or objects which already have a sign: the Bear (Great or Little), Swan, Fish, Whale, etc. Mythological names follow the traditions of legend; thus Orion is a hunter, and the Centaur is a being with the torso of a man mounted on the body of a horse.

Technology is signed in a similarly way, for example COMPUTER which is defined in sign language according to its type (PC, portable, etc). Some terms often have a discreet sign like a Numeric which becomes 1-0-1-0-1-0. Similarly, the worlds of medicine and biology have very complete and technical vocabularies.

This rapid overview can obviously only give a bare outline of scientific communication in sign language. Facial expression is of great importance whether its to express a mathematical series tending to infinity i.e. very small, or that the star Vega in the constellation of Lyra has a surface temperature of 35000 degrees, i.e. very hot. In addition to the precision of a scientific discourse, the signer accompanies his talk with gestures in which the series of signs depends on interpretation in the musical sense of the term. This interpretership/interpretation duality turns a talk in the strict sense into a talk that is not only understood but felt as well. In this way, the association of bodily expression with academic tradition adds a touch of humanity and sharing in a somewhat rough world. 

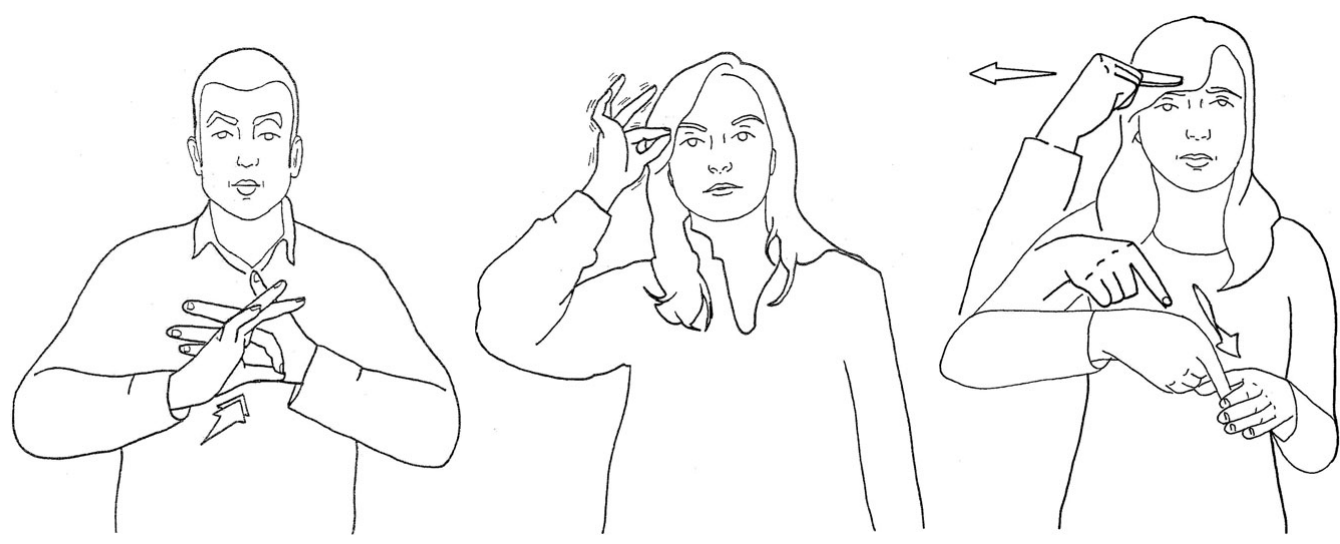

Figure 3. From left to right, how to sign Jupiter (red spot), Star, Black Hole (hole + black).

\section{An LSF dictionary of astronomy}

The idea for a dictionary of astronomy saw the light of day following a broadcast in LSF of the television series $L$ Oeil et la Main (Eye and Hand) which was devoted to this science, produced by Daniel Abbou, with the assistance of the authors of this dictionary and broadcast in October 2007 (http://www.france5.fr/oeil-et-main/archives/ 35220934-fr.php). Furthermore, since 2000, there have been monthly classes in astronomy organised by the Observatory of Paris-Meudon in the framework of the series Astronomy for Everyone, the aim of which has been to make known astronomy, astrophysics and related sciences (planetology, climatology, exobiology) to a public that has had difficulty in accessing scientific culture (including hospitals, prisons, etc.). This training brings twelve to fifteen deaf persons together for each session. If weather conditions permit, observations are carried out with one of the observatorys telescopes having first described the chosen objects (Moon, planets, stars, galaxies, etc.) and then observing them in the darkness. If the sky is overcast, besides visiting the observatory, a themed conference takes place with suitable visual aids. These meetings are much appreciated by the deaf community and they allow particularly fruitful exchanges which go beyond a formal scientific framework and from which everyone gains something. For the presenter, they are an opportunity to learn more about the world and culture of the deaf as well as getting to grips with the practice of sign language. This experience has resulted in this dictionary.

This dictionary is the first to establish a link between astronomy and the deaf community. If astronomy is one of the oldest sciences, the difficulty of mans understanding of an immense universe, in which space and time both come into play, runs in parallel with the concerns that the deaf have in a noisy world. So the signs for essential terms in astronomy have resulted in the creation of neologisms and most notably for terms borrowed from tradition. As an example, finding an equivalent sign for the names of the constellations is easy where animals and items are concerned whereas the names of constellations that go back to the time of Ptolemy and Ancient Greece require greater imagination. Cepheus is represented by a compound sign bearded King, Cassiopeia by the sign the Queen, and their daughter Princess Andromeda by the compound sign the bound woman which refers to the myth which shows her bound to a rock after having incurred Poseidons wrath.

We have been careful to avoid homonyms or paronyms; for example it was essential to avoid confusion between Saturn with its thin rings and a galaxy with its arms. This search for equivalent signs has given rise to much thought when the astronomical term 

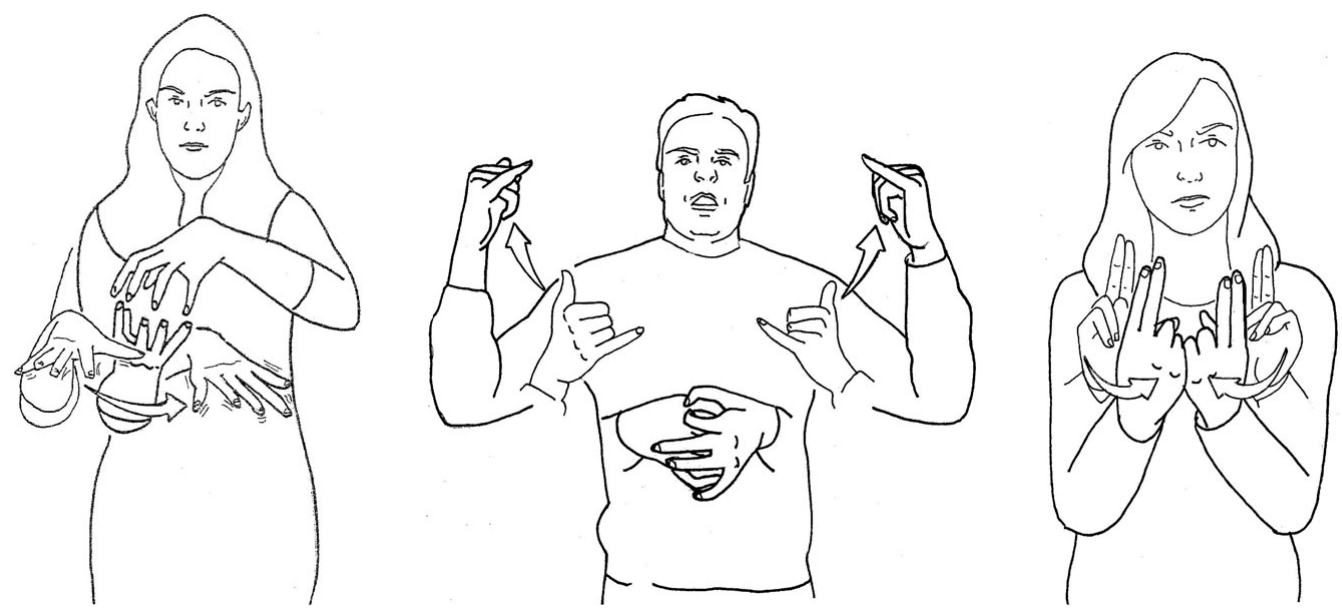

Figure 4. From left to right, how to sign Galaxy, Quasar (galaxy + quasar), Universe.

only appeared recently and refers to objects of great complexity. A typical example concerns the word QUASAR which is a contraction of the term quasi stellar radiosource. We had to wait until the 1960s to understand that a quasar is not a star, even though it seems to have the same dimensions as one, but is a much more distant object whose energy of radiation, as great as any galaxys, is contained in a very small core. A signed description put forward by the deaf colleagues of this dictionary is as follows: I see a small but brilliant source in the sky; I open it to see its interior; I am astonished to observe the central region of a galaxy confined in this space with its incredible energy. This translates into the compound sign: SELF + GALAXY + ENERGY EMITTED + POWERFUL.

We have to wait for these constructions to evolve in the hands of the deaf, either because new knowledge will allow better semiological adaptation or because, like all living languages, sign language tends to be modified over time generally by reducing complicated signs to something more simple. We are the first to wish for such an evolution. This dictionary has as its aim to bring together the essentials of astronomy and to convert them into sign language which is where this encyclopaedic form comes from. For the reader who doesnt use sign language, a basic course should be followed in parallel. This can be obtained from various bodies and associations and is generally given by deaf teachers. We have wanted to create a tool as much for the teachers as for those who are interested in deaf culture and astronomy. Each entry is accompanied by a picture of the corresponding sign as well as a commentary which states precisely what the different parameters of each sign symbolise. When the sign refers to something ancient, this commentary also gives a etymological overview. The pictures are the result of Carole Marions talents; movement is shown by arrows in line with conventions long established. Reference of this dictionary is given below.

\section{References}

Proust, D (sous la direction de), Abbou, D., Chab, N., Delaporte, Y., Marion, C., \& Proust, B. 2009, Les Mains dans les Etoiles, dictionnaire d'astronomie pour la LSF, (Vannes: Editions Burillier) 